\section{ANTI-R052 EPITOPE MAPPING IN INFLAMMATORY MYOPATHIES}

Olga Krystufkova, ${ }^{1}$ Vijole Dzikatite, ${ }^{1}$ Peter Charles, ${ }^{3}$ Herman F Mann, ${ }^{1}$ Louise Ekholm, ${ }^{1}$ Melinda Vincze, ${ }^{2}$ Ivana Putova, ${ }^{2}$ Nikola Kasprikova, ${ }^{4}$ Peter Novota, ${ }^{4}$ Leonid Padyukov, ${ }^{1}$ Katalin Danko, ${ }^{2}$ Jiri Vencovsky, ${ }^{2}$ Marie Wahren-Herlenius, ${ }^{1}$ Ingrid E Lundberg' ${ }^{1}$ Rheumatology Unit, Department of Medicine, Karolinska University Hospital, Solna, Karolinska Institutet, Stockholm, Sweden; ${ }^{2}$ Division of Clinical Immunology, 3rd Department of Medicine, University of Debrecen, Debrecen, Hungary; ${ }^{3}$ Kennedy Institute, London, UK; ${ }^{4}$ nstitute of Biophysics and Informatics, 1 st Faculty of Medicine, Charles University Prague, Prague, Czech Republic

10.1136/annrheumdis-2011-201235.19

Background Specific or associated autoantibodes are present in a majority of patients with idiopathic inflammatory myopathies (IIM). The most frequent myositis associated autoautoantibodies are directed to Ro52-kd protein (present in
$12-26 \%)$. Antibodies towards the immunodominant epitope (p200) of Ro52 are associated with neonatal heart block (CHB) in SLE and Sjögren's syndrome $(\mathrm{SjS})$ with the increasing evidence for their pathogenic role. Less is known about possible pathogenic involvement of anti-Ro52 in IIM. Presence of antiRo52 in patients with anti-Jo-1 autoantibody is associated with interstitial lung disease (ILD) and suggested decreased survival rate.

Objective To investigate possible associations of reactivity to Ro52 epitopes with disease manifestations, autoantibody profile and HLA-DRB1 alleles in myositis. In order to confirm the hypothesis of ongoing epitope spreading with time, the association of disease duration with polyreactivity was evaluated. Methods Serum samples from 468 myositis patients were collected in three countries: Czech $(n=133)$; Hungary $(n=202)$, Sweden ( $n=133$ ). Antibody specificity against recombinant full length (FL) Ro52 antigen, its four deletion fragments (Ro52-3, $-4,-5$ and -6 ) and 7 overlapping synthetic peptides (p136-200) covering the major immunodominant region (Ro52-3) was measured by ELISA. Myositis specific and associated autoantibodies (Jo-1, PL-7, PL-12, EJ, OJ, SRP, PM-Scl, Ku, Mi-2 $\beta$, U1-RNP) were detected by line-blot immunoassay. 417 patients were genotyped for HLA-DRB1.

Results One hundred and eighty-four patients with dermatomyositis (DM), 250 polymyositis (PM), 11 inclusion body myositis, 20 juvenile DM/PM and three with mixed connective tissue disease were included in the analysis. Overlap with other systemic disease (OM) was present in $67(14 \%)$ and ILD in $132(29 \%)$ of patients. Median disease duration was 4.7 $(0-41.5)$ years.

Reactivity to Ro52-FL was present in 97 (21\%) samples. Of these, $87(90 \%)$ patients showed specificity to Ro52-3. Within these anti-p176 dominated (66; 76\%) compared to anti-p200 in $44(51 \%)$ patients.

Anti-Ro52FL positivity was associated with presence of ILD (OR 3.8; $\mathrm{p}<0.0001)$, anti-Jo1 (OR=9.0; $\mathrm{p}<0.0001)$ and HLADRB1*03 allele $(\mathrm{OR}=1.9 ; \mathrm{p}=0.009)$. No significant epitope associations were detected. The reactivity to 2 or more peptides was associated with $\mathrm{OM}(\mathrm{p}=0.036)$. No correlation with disease duration was found.

Conclusion Our results confirm the association between antiRo52 autoantibody positivity with lung involvement, anti-Jo-1 autoantibodies and HLA-DRB1*03 allele in myositis.

The predominance of reactivity to epitope p176 in myositis patients is different from that which has been reported in patients with SLE and SjS, but the clinical significance is not clear. There was no evidence of epitope spreading in anti-Ro52 response.

Acknowledgement Supported by European Union 6.FP integrated project AutoCure LSHB CT-2006-018661; by MSM 0021620812 from Ministry of Education, Youth and Sports in the Czech Republic and by Grant NT 12438 of IGA Ministry of Health in the Czech Republic. 